\title{
Clinical and laboratory evaluation of the physical properties of two light cured fissure sealants
}

\begin{abstract}
Aim: To use and validate a new indirect area mapping technique which allows differentiation between peripheral wear and other modes of peripheral bulk loss of fissure sealants in clinical service. To measure wear and the other modes of loss of 2 different unfilled visible light polymerized Bis-GMA resins based fissure sealants over a 4 year period.
\end{abstract}

Materials and methods: Helioseal and Delton Opaque fissure sealants were applied to pairs of contralateral mandibular second premolar teeth. Optically clear epoxy resin replicas were produced which were sequentially located and mounted within a defined plane. The replicas were transilluminated from below with diffused light, examined with a microscope and digital images captured. This allowed the entire fissure sealant peripheral border to be clearly identified and mapped. Categorized mapped areas were measured.

Results: The technique allowed the accurate measurement of the area of peripheral material loss attributed to wear and the other modes of loss which involved sealant-enamel interface failure, these peripheral bulk losses were classified as either internal or external to the peripheral recession attributed to wear. Over the 4 year trial period more than $99 \%$ of the area of sealant loss was attributed to wear. At baseline the mean area of the Helioseal and Delton Opaque fissure sealants was $9.97 \mathrm{~mm}^{2}$ and $7.76 \mathrm{~mm}^{2}$ respectively. At 2 years the mean area of Helioseal had become fractionally smaller than that of Delton Opaque. The increased rate of wear of Helioseal compared to Delton Opaque continued from 2 through to 4 years, this difference being statistically significant $(\mathrm{P}<.001)$. Irrespective of the material applied, after the anticipated initial phase of rapid wear, the rate of peripheral wear was progressive and did not plateau.

Conclusion: The technique was validated. The area of fissure sealant loss was demonstrated to be almost exclusively attributed to wear. The rate of wear of Helioseal was greater than for Delton Opaque. For both materials, after the initial phase of rapid wear, the wear was demonstrated to be progressive.

Keywords: clinical, laboratory, wear, sealants

\author{
Volume 7 Issue I - 2017
}

\author{
Donnan MF,' Gray GB, ${ }^{2}$ Vowles RW ${ }^{3}$ \\ 'Retired Specialist in Pediatric Dentistry, University Hospitals \\ Bristol Primary Care Dental Service, UK \\ ${ }^{2}$ Retired Dental Clinical Dean, University of Bristol Dental \\ School, UK \\ ${ }^{3}$ Retired IT Support Specialist, Computer Services, University \\ of Bristol, UK
}

\begin{abstract}
Correspondence: Donnan MF, Operational Manager, University Hospitals Bristol Primary Care Dental Service, University of Bristol Dental Hospital, Lower Maudlin Street. Bristol BSI 2LY, UK, Tel 44 II 7342 9583, Email Kay.Paul@uhbristol.nhs.uk
\end{abstract}

Received: March 04, 2017 | Published: April 17, 2017
Abbreviations: 3D, three-dimensional; 2D, two-dimensional; SEM, scanning electron microscopy; Bis-GMA, bisphenol-Aglycidyl-dimethacrylate resin; IPL, internal peripheral loss not attributed to wear; EPL, external peripheral loss not attributed to wear

\section{Introduction}

Resin based fissure sealants are considered to be effective in preventing caries as long as they are retained and occlude the pits and fissures. ${ }^{1}$ The caries reduction in pits and fissures from the use of sealants on permanent teeth has been demonstrated in numerous clinical studies. ${ }^{2}$ Fissure sealant loss has been considered to occur in one of two ways: either due to gradual wear or due to bulk loss of all or part of the sealant, this being attributed to bonding failure and associated with salivary contamination. ${ }^{3}$ Early loss of sealants in clinical studies has been attributed to application factors, in particular by allowing moisture or salivary contamination. ${ }^{4,5}$ Over the years, there have been a number of reports where methods have been used to assess or measure the amount of applied fissure sealant material. These reports have described sealants assessed by direct observation, ${ }^{6}$ by direct photographic techniques, ${ }^{7,8}$ and by photographing silicone impressions $^{7}$ and epoxy casts. ${ }^{9}$ The volume of fissure sealants has been measured using a vinyl-epoxy resin die and silver coping technique. ${ }^{10-12}$ Volume, depth and three-dimensional (3D) surface area have been measured by using epoxy resin replicas with a displacement stylus coupled with computer graphics. ${ }^{13-17}$ The two-dimensional (2D) surface area of sealants has been measured using gold plated epoxy resin replicas and a photographic mapping technique. ${ }^{18,19}$ More recently, the cross sectional area of sealants has been measured using sliced silicone impressions and scanning electron microscopy (SEM).$^{20}$ None of the above measuring techniques, however, differentiate between material losses due to wear and bulk loss involving failure at the sealant-enamel interface. The aim of the present study was therefore to use and validate a new indirect technique that includes a microscopic examination and digital tracing of the entire fissure sealant peripheral border to enable both types of material loss to be identified and measured. This technique was used in a 4-year clinical trial to measure these modes of peripheral material loss of the light cured unfilled Bisphenol-A-glycidyl-dimethacrylate (Bis-GMA) resin based fissure sealants Helioseal and Delton Opaque. 


\section{Materials and methods}

Ethical approval was granted for the clinical trial (Research Ethics Committee, United Bristol Healthcare NHS Trust, and United Kingdom) with informed consent being received. Forty patients aged between 10 and 19 were selected for the trial from patients who regularly attended a Community Dental Service clinic in North West Bristol or Bristol Dental Hospital Community Dental Unit. The fluoride level in the drinking water throughout the trial was 0.2 parts per million. The inclusion criteria for each participant stipulated the presence of contra lateral mandibular second premolars of similar morphology in standard occlusion with no observed defects or hypo mineralization. In addition, the teeth needed to be judged clinically and radiographically caries free. At a preliminary appointment, alginate (Alginoplast; Bayer Dental, Leverkusen, Germany) impressions were taken and used to produce plaster study casts which were subsequently used to check the occlusion and for the laboratory location technique described later. At the following baseline appointment, a random numbers table was used to determine which of the contra lateral teeth was to be sealed first and with which material. Either Helioseal (Batch number: 440128. Ivoclar Vivadent, Schaan, Liechtenstein) or Delton Opaque (Batch number: 931123. Dentsply, Weybridge, United Kingdom) was used. Each study tooth was isolated with a saliva absorber (Dry Guards; Virilium, Watford, United Kingdom) and a flange saliva ejector and then thoroughly dried with compressed air. The tooth was then etched with $35 \%$ phosphoric acid liquid (Dentsply) for 60 seconds. This was followed by a wash with water and compressed air spray with aspiration for 10 seconds. The tooth was then further isolated with cotton wool rolls and again thoroughly dried with compressed air. The allocated fissure sealant was incrementally applied with a spot spatula (Dycal spot spatula; Dentsply) aiming to apply a similar amount for each material. The flat tip of a light curing unit wand (Translux tungsten-halogen visible light curing unit; Kulzer, Hanau, Germany) was held for 40 seconds within $1-2 \mathrm{~mm}$ of the occlusal surface. There was no occlusal adjustment of the sealant. Plastic mini trays $2 \mathrm{~cm}$ wide and $2.5 \mathrm{~cm}$ long with inwardly angled lateral edges with greenstick composition dams at either end were used for the impressions of the study sealants. The composition dams were warmed at the chair side with a hot air heater (Safe Air; Carbolite Furnaces, Sheffield, United Kingdom) and the tray pre-seated onto the teeth adjacent to the study tooth to produce locating and spacing stops. The study tooth was then isolated as above and the sealed occlusal surface thoroughly wiped with a small tightly compressed cotton pledget soaked in absolute alcohol and held in tweezers. The sealed occlusal surface was then thoroughly dried with compressed air. A putty and wash addition polymerized silicone impression (Elite putty and light body; Zhermack, Badia Polestine, Italy) was taken using a single stage technique. These impressions were taken at baseline, 6 months and then annually up to 4 years. A tolerance at a ratio of 1 month either side of the baseline anniversary was allowed for each year elapsed in the trial. An optically clear epoxy resin (Araldite MY 753 with XD 716 hardener; B and K Resins, Bromley, United Kingdom) was used to produce replicas from the silicone impressions. The plane in which the replica of the sealed tooth was to be subsequently viewed was defined by 3 points on the mandibular study cast. These were the highest points of the two first molars and one of the first premolar teeth. This plane of orientation was produced by using a microscope slide covered on one side with silicone putty (Extrude VPS putty; Kerr, Peterborough, United Kingdom) which was seated onto the cast so that these 3 highest points were seen contacting the glass through the putty. The occlusal surface of the pre-sealed mandibular second premolars on the plaster study cast had been spaced in order that this putty impression would subsequently accommodate the replica baseline sealant. This silicone putty impression was then used in a jig to locate and initially mount the baseline replica in that orientation. The initially mounted baseline replica was then removed from the jig. The silicone putty impression slide was replaced by a new slide onto which newly mixed zinc oxideeuginol impression paste (SS White, Gloucester, United Kingdom) had been placed. The initially mounted baseline replica, having been sprayed with artificial saliva (Glandosane Spray; Fresenius-Kabi, Runcorn, United Kingdom) as a separating medium, was re-seated in the jig. This produced a zinc oxide-euginol locating matrix for that series of replicas. Before final mounting, the fit of each replica within the matrix was verified using a light source and magnification to confirm the absence of internal reflections at the interface of the optically clear replica and the zinc oxide-euginol locating matrix (Figures 1A \& 1B). A replica being mounted is illustrated (Figure 1C). The accuracy of location and mounting for each of the replicas within a given series was verified using a profile projector with an extremely limited depth of field (Profile Projector 6CT2; Nikon, Kingston upon Thames, United Kingdom). This was used to identify 3 triangulating features in focus on the baseline replica (Figure 1D). The rest of the mounted series were viewed to ensure that these triangulating features were in focus thus verifying their correct location. Any failures were investigated, the fault corrected, the replica remounted and the check repeated. A digital camera (DS-505; Fuji, Tokyo, Japan) with cable release was fitted to a binocular microscope (Nikon SMZ-U; Nikon). The microscope base had been modified by fitting a disk of opaque plastic film (Parafilm M; Pechiney Plastic Packaging, Chicago, USA) into the light aperture as an additional illumination diffuser. The light from the source within the base of the microscope was reflected upwards using the diffusing side of the internal mirror and adjusted to optimise the definition of the viewed image of the transilluminated replica. External light sources had been turned off. The series of sequentially mounted replicas were photographed. Initially a photograph was taken of a graticule (Athene Grid Type 110; Agar Scientific, Stansted, United Kingdom) which had been lowered onto the first replica in each series. This image was used later to provide a scale for calibration. The digital image of each replica of the sealed tooth in each series was then captured and saved.

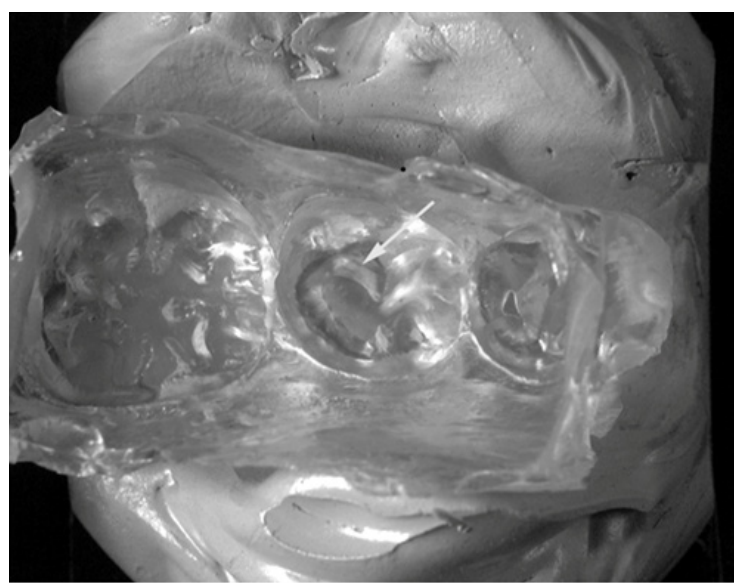

Figure IA Internal reflections due to gap at interface of replica and zinc oxide-euginol locating matrix revealing inadequate location are indicated by arrow. 


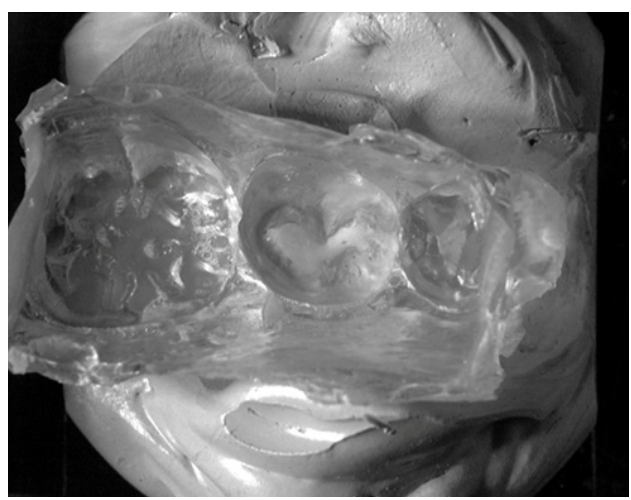

Figure I B Absence of internal reflections reveals correct location.

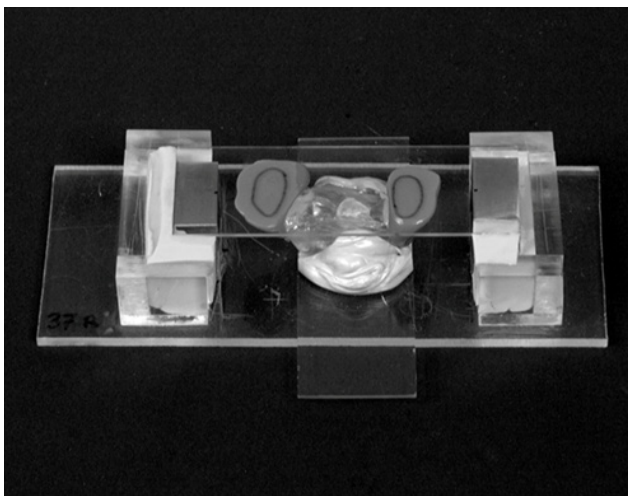

Figure IC Replica has been located in zinc oxide-eugenol matrix and is being mounted on silicone fins in order to allow subsequent transillumination.

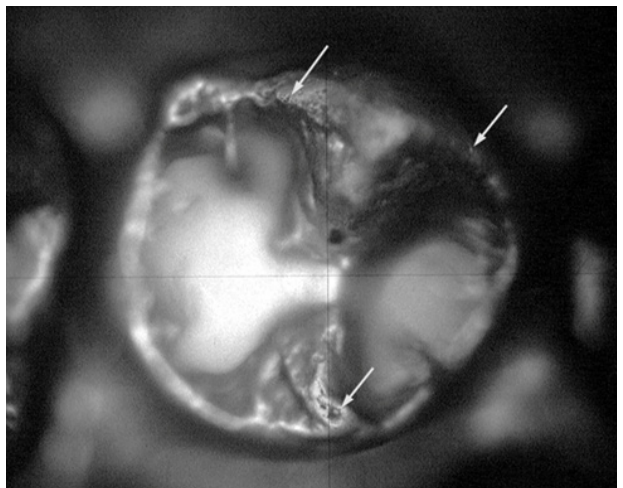

Figure ID Three triangulating features in focus within limited depth of field of profile projector confirm accurate location and mounting.

The captured image of each replica in the series was subsequently examined on a computer monitor with reference to the actual replica viewed through the binocular microscope. The replica was initially examined to check if the peripheral border of the sealant was still located on the cuspal inclines and whether there was any exposure of the pit or fissure embrasure. The next stage involved examining sections of the peripheral border of the replica fissure sealant via the binocular microscope and tracing each section on the corresponding computer image. The tracing was carried out at a width of 1 pixel using a digitising pen and tablet (Graphics Tablet; Wacom, Saitama, Japan) and a graphics editing program (Paint Shop Pro 3.0; Jasc Softwear, Minesotta, USA). This process was repeated until the entire peripheral border of each sealant image had been traced (Figure 2A). In order to establish whether or not the recession of the sealant periphery was broadly even between each time interval of the study a computer split screen was used to view the sequence of tracings. For any given comparison, if any parts of the traced peripheral border did not follow the outline of the preceding tracing the replicas were examined to confirm that at any such site the periphery exhibited an edge consistent with being caused by bulk loss. These areas were recorded as peripheral loss not attributed to wear. Peripheral losses not attributed to wear could either be due to a portion being lost from within the body of the fissure sealant which involved the peripheral border or be due to an extension of the fissure sealant becoming detached. The former type of bulk loss was classified as an Internal Peripheral Loss not attributed to wear (IPL) (Figure 2B) and the latter as an External Peripheral Loss not attributed to wear (EPL) (Figures $2 \mathrm{C} \& 2 \mathrm{D})$. The area of these losses was traced using the graphics pen. The area of the IPL was traced on the image of the loss allowing the area of the enamel exposed to be directly measured. The area of the EPL was traced on the preceding image and the measurement then adjusted in line with the overall rate of sealant area loss by multiplying the measurement by the value derived from the sealant area when the loss had occurred divided by the preceding sealant area. The area encompassed by the tracings was calculated with an image analysis program (Imaging Primer 1.2; Graftek Imaging, Austin, Texas, USA) which had been calibrated with the graticule image. Four of the sets of paired images were randomly allocated and hidden within the coded sets given to the operator to allow a repeatability analysis.

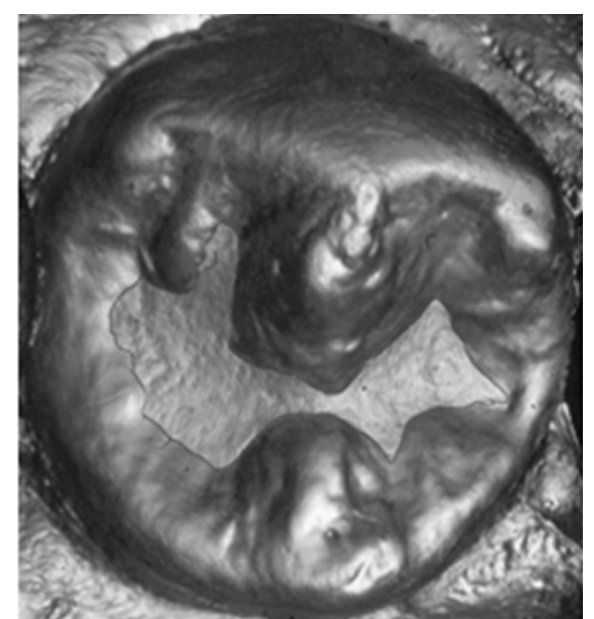

Figure 2A Fissure sealant peripheral border has been traced with one pixe width line.

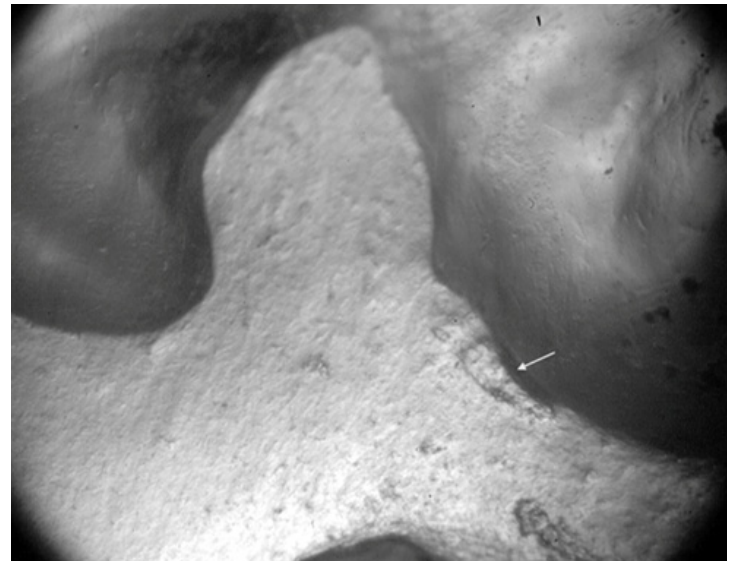

Figure 2B Internal peripheral loss not attributed to wear (IPL) indicated by arrow. 


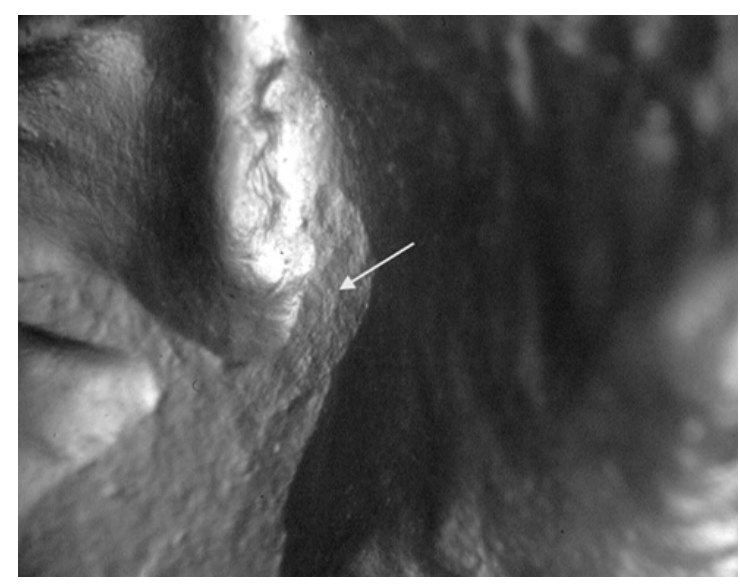

Figure 2C Portion of fissure sealant which would be detached by next review indicated by arrow.

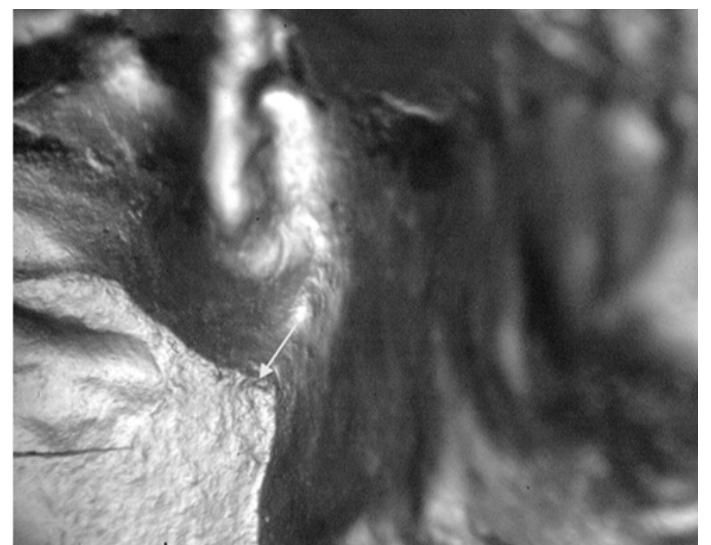

Figure 2D External peripheral loss not attributed to wear (EPL) has occurred. Characteristic edge where portion has detached from main body of fissure sealant indicated by arrow.

\section{Results}

Forty pairs of mandibular second premolars were included at baseline, 39 (98\%) were reviewed after 6 months, 39 (98\%) after 1 year, $38(95 \%)$ after 2 years, $33(83 \%)$ after 3 years and $32(80 \%)$ after 4 years. The 32 pairs reviewed at 4 years had also been reviewed at every preceding interval and therefore provided complete sets of measurements for the same 32 matched pairs. The mean depth of field of the profile projector used to verify the location and mounting of the replicas was calculated to be $34.5 \mu \mathrm{m}$. On 10 occasions $(2.26 \%)$ out of 442 mounting episodes, the depth of field technique identified a failure in accurate location. In each situation the fault was immediately identified as being either due to human error during the mounting process or as a result of interference in the fit by an epoxy resin extrusion. On correction of the fault, all 10 replicas passed the depth of field accuracy of location check. The calibration from the 80 digital images of the graticule also allowed the pixel size to be calculated at a mean of $8.5 \mu \mathrm{m}$ with a standard deviation of $1.0 \mu \mathrm{m}$. The four sets of fissure sealant pair images which were re-measured blindly produced repeat measurements. For the calibrating image; 5 of the 8 repeat graticule measurements were identical; the mean of the 3 where there was a difference was $0.20 \%$. The mean difference in the mapped sealant area for the repeated measurements was $2.24 \%$ at baseline, $0.82 \%$ at 6 months, $0.92 \%$ at 1 year, $1.82 \%$ at 2 years, $1.90 \%$ at 3 years and $1.72 \%$ at 4 years. The examination of the transilluminated replicas with the binocular microscope revealed that for all of the reviewed study teeth, the sealant peripheral border remained on the cuspal inclines and there was no exposure of a pit or fissure embrasure. All the sealants were therefore fully retained. The measurements for the complete set of the same 32 matched sealant pairs which continued through to the end of the trial were analysed. The area of the 2 modes of peripheral loss not attributed to wear (IPL and EPL) and the combined area (IPL+EPL) for Helioseal and Delton Opaque are presented as a percentage of the area loss between the intervals and as an overall percentage in Table 1 . The mean area measurements for Helioseal and Delton Opaque at baseline and the subsequent reviews are presented in Table 2 \& Figure 3.

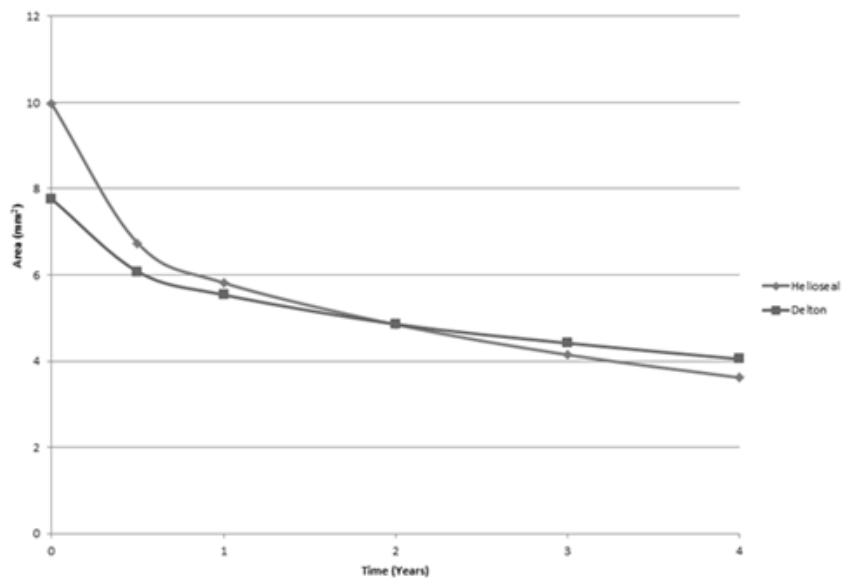

Figure 3 Mean area measurements of 32 Helioseal and Delton Opaque pairs.

Table I Internal (IPL), external (EPL) and combined (IPL+EPL) peripheral material loss not attributed to wear

\begin{tabular}{|c|c|c|c|c|c|c|c|}
\hline \multirow{2}{*}{\multicolumn{2}{|c|}{ Number of pairs }} & \multirow{2}{*}{\multicolumn{2}{|c|}{$\frac{\text { IPL }}{\text { Helioseal Delton opaque }}$}} & \multirow{2}{*}{$\begin{array}{l}\text { EPL } \\
\text { Helioseal }\end{array}$} & \multirow{3}{*}{$\begin{array}{l}\text { Delton opaque } \\
0.77 \%\end{array}$} & \multicolumn{2}{|l|}{ IPL+EPL } \\
\hline & & & & & & \multirow{2}{*}{$\begin{array}{l}\text { Helioseal } \\
0.18 \%\end{array}$} & \multirow{2}{*}{$\begin{array}{l}\text { Delton opaque } \\
0.82 \%\end{array}$} \\
\hline Six months & 32 & $0 \%$ & $0.05 \%$ & $0.18 \%$ & & & \\
\hline One Year & 32 & $0.07 \%$ & $0.57 \%$ & $2.23 \%$ & $0 \%$ & $2.30 \%$ & $0.57 \%$ \\
\hline Two Years & 32 & $0.16 \%$ & $0.45 \%$ & $0.03 \%$ & $0.72 \%$ & $0.19 \%$ & $1.17 \%$ \\
\hline Three Years & 32 & $0.37 \%$ & $0.36 \%$ & $0.30 \%$ & $0.53 \%$ & $0.67 \%$ & $0.89 \%$ \\
\hline Four Years & 32 & $0 \%$ & $0 \%$ & $0.45 \%$ & $0 \%$ & $0.45 \%$ & $0 \%$ \\
\hline Overall loss & 32 & $0.08 \%$ & $0.23 \%$ & $0.49 \%$ & $0.54 \%$ & $0.57 \%$ & $0.77 \%$ \\
\hline
\end{tabular}


Table 2 Mean area measurements of the same 32 Helioseal and Delton Opaque pairs

\begin{tabular}{|c|c|c|c|c|c|c|}
\hline & \multirow{2}{*}{ Number of Pairs } & \multicolumn{3}{|l|}{ Helioseal } & \multicolumn{2}{|l|}{ Delton Opaque } \\
\hline & & Mean area $\mathbf{m m}^{2}$ & Standard de & viation & Mean area $\mathbf{m m}^{2}$ & Standard deviation \\
\hline Baseline & 32 & 9.97 & 3.03 & & 7.76 & 2.32 \\
\hline Six Months & 32 & 6.74 & 2.15 & & 6.08 & 1.69 \\
\hline One Year & 32 & 5.82 & 1.94 & & 5.54 & 1.6 \\
\hline Two Years & 32 & 4.85 & 1.65 & & 4.86 & 1.43 \\
\hline Three Years & 32 & 4.15 & 1.42 & & 4.42 & 1.39 \\
\hline \multirow[t]{4}{*}{ Four Years } & 32 & 3.62 & 1.31 & & 4.05 & 1.36 \\
\hline & & \multicolumn{2}{|l|}{ Helioseal } & \multicolumn{2}{|c|}{ Delton opaque } & \\
\hline & & \multicolumn{2}{|c|}{ Mean area reduction $\mathbf{M m}^{2}$} & \multicolumn{2}{|c|}{ Mean area reduction $\mathbf{M m}^{2}$} & \\
\hline & $2-4$ years & 1.23 & & 0.81 & & \\
\hline
\end{tabular}

The t-Test was applied to test the differences between the area reductions of the 32 matched pairs of Helioseal and Delton Opaque fissure sealants from 2-4 years. The differences were significant $(\mathrm{P}<.00 \mathrm{I})$

\section{Discussion}

The aim of this study was to use and validate a technique that would allow the microscopic examination and tracing of the complete peripheral border of fissure sealants and would differentiate between and measure both fissure sealant ware and peripheral bulk loss (IPL and EPL). This is in contrast to previous techniques used to measure sealants which have not taken into account peripheral bulk loss of material. ${ }^{10-20}$ The dimensions of a fissure sealant can be represented by volume or surface area. When a fissure sealant wears, tooth surface is exposed by loss of material at its peripheral border. Some workers have measured fissure sealant volume, ${ }^{10-12}$ however, because a sealant can be considered as a conical mass of material within the cuspal inclined planes this will result in a skewed representation of tooth surface exposure. The 3D surface area of fissure sealants has been measured in conjunction with volume. ${ }^{13-17}$ If a sealant's surface area is measured in 3D, there can also be misrepresentation of tooth surface exposure. Profiles illustrate that with wear the fissure sealant surface can become increasingly concave ${ }^{17}$ resulting in an under estimation of the tooth surface exposed. A 2D peripheral mapping technique has been used. ${ }^{18,19}$ In both of these studies, photographs produced from gold plated epoxy replicas successfully represented tooth surface exposure. A simpler 2D peripheral mapping technique can provide a more direct representation of tooth surface exposure as the sealant peripheral border recedes down the cuspal inclined plane. At a very late stage, if the sealant has receded into the fissure embrasure, such a technique would then become redundant as the angulation changes and becomes more vertical. Mandibular second premolar teeth were selected for the present study because of their ease of access and because they have an open cuspal morphology. Teeth with a more open and flatter anatomy expose a greater area of tooth surface for a given volume loss,${ }^{17}$ thus allowing wear with peripheral recession and tooth surface exposure to be well represented by a mapping technique. With any 2D mapping technique, the accuracy of the sequential location of the replicas in a given series is critically important if inaccuracy in measurement is to be avoided. Previous studies have reported the sequential location of fissure sealant replicas. ${ }^{18,21}$ The methodologies described were explored as part of the development of the technique used in the present study but rejected because they were found to be technically very difficult. In the present study, the accurate location of each replica in the matrix was initially verified before mounting. This proved straightforward to achieve by simply noting an absence of internal reflections at the interface of the optically clear replica and the locating matrix (Figure 1B). After mounting, the accurate location of the replicas in the series was confirmed by the triangulated features being in focus within the extremely limited depth of field of the profile projector (Figure 1D). During the development of the technique, silicone impression material was tried as the locating matrix for the mounting of the replicas. Silicone, however failed to allow the accurate sequential location of replicas. This failure was obvious because when the initial location check was carried out internal reflections were invariably observed with the series of replicas subsequent to baseline, the baseline replica having been used to produce the locating matrix. This failure was attributed to small differences in the surface of the elastic silicone matrix or the rigid resin replica, causing rebound therefore resulting in an inaccurate fit. The solution to this problem was to take advantage of the particular properties of set zinc oxide-eugenol impression paste. This material has the overall rigidity required to hold the replica but also has an element of accommodation due to its plasticity under load. Its surface allows the flattening of any positive matrix interferences while also allowing any positive replica interferences to penetrate, so allowing replicas subsequent to baseline to fit. Successful location was confirmed by the absence of internal reflections at the check. The only difficulty encountered was that during fabrication of the locating matrix, the newly mixed paste adhered to the baseline replica. This problem was overcome by applying a thin layer of artificial saliva to the baseline replica which acted as a separating medium. The technique used in the study for the sequential location and mounting of each series of replicas with a zinc oxide-eugenol matrix proved to be extremely accurate. All the satisfactorily mounted replicas, when checked with the profile projector, were demonstrated to be located to a greater degree of accuracy than its depth of field which was calculated to be $34.5 \mu \mathrm{m}$. In addition it proved very reliable with only just over $2 \%$ of the mounted replicas requiring fault correction and remounting. A mean pixel size of $8.5 \mu \mathrm{m}$ demonstrated the detail of the captured digital images. The repeat measurements of 4 pairs of the image sets demonstrated that the image calibration from the graticule 
was very accurate. When the sealant area tracings were repeated the greatest difference, at $2.24 \%$, was found between the measurements of the baseline sealant. This was attributed to the peripheral border being less well defined. The greater difference between the repeat measurements, at $1.82 \%, 1.90 \%$ and $1.72 \%$ for 2,3 and 4 years respectively compared to those at $0.82 \%$ and $0.92 \%$ for 6 months and 1 year respectively was attributed to being a function of the reduced surface area being measured. The initial microscopic examinations revealed that the peripheral border of all of the sealants remained on the inclined planes with no exposure of pit or fissure embrasure. Consequently, this 2D mapping technique was not compromised by any change in angulation associated with an inclined plane steepening at the embrasure. In addition, all of the sealants were classified as fully retained. Indirect microscopic examination of retention has been demonstrated to be a more critical evaluation than direct clinical visual assessment. ${ }^{22}$ The enamel surface angulation differed according to the tooth's morphology and, as expected, it was noted that there was a correspondingly greater rate of peripheral recession represented in the flatter areas. Typically the receded peripheral border was less well defined in these flatter areas than on the steeper inclines. This varied appearance of the peripheral border noted in the present study concurs with previously reported SEM observations were the material loss was attributed to abrasion. ${ }^{9}$ The present technique allowed both the differentiation between, and measurement of, the area of loss attributed to wear at the periphery of the sealant and the area of bulk loss involving failure at the sealant-enamel interface. When expressed as a percentage of the total peripheral area loss for the same 32 pairs of sealants, the area of peripheral loss not attributed to wear internal to the peripheral recession (IPL) over the 4 year duration of the clinical trial gave values of $0.08 \%$ and $0.23 \%$ for Helioseal and Delton Opaque respectively. A distinct edge circumscribed these areas of loss (Figure 2B). This would suggest both a cohesive failure within the material itself and failure at the enamel interface. A similar appearance has been noted from SEM observations in a study which included a BisGMA based sealant with $6 \%$ filler loading. ${ }^{23}$ The corresponding values for peripheral loss not attributed to wear external to the peripheral recession (EPL) accounted $0.49 \%$ and $0.54 \%$ for Helioseal and Delton Opaque respectively. A characteristic edge was invariably present where an external peripheral loss had occurred. This appearance is suggestive of failure at the enamel interface leading to a detachment of that portion of sealant from the main body so producing an edge (Figures 2C \& 2D). The measurements for the IPL and EPL were combined to produce a percentage area of material lost at the periphery not attributed to wear. Over the 4 years in clinical service the percentage of the area loss which was not attributed to wear was $0.57 \%$ and $0.77 \%$ for Helioseal and Delton Opaque respectively. Therefore, the peripheral loss of material of these unfilled Bis-GMA based sealants can be attributed almost exclusively to wear processes. At baseline, for the 32 pairs of sealants which continued through every review to the completion of the clinical trial, the mean area of Helioseal was $9.97 \mathrm{~mm}^{2}$ which was greater than Delton Opaque at $7.76 \mathrm{~mm}^{2}$. Despite using a specific technique endeavouring to apply a similar amount of material, a greater coverage had occurred with Helioseal. This may be explained by the different handling characteristics of the 2 materials, with increased wetting from the lower viscosity Helioseal. As anticipated, for both materials, the rate of area loss was greatest from baseline to 6months. Of particular interest was the continued and progressive rate of loss from 2 through to 4 years (Figure 3 ). At 2 years, despite having a greater mean area at baseline, the area of Helioseal become fractionally smaller than that of Delton Opaque. This increased rate of wear of Helioseal compared to Delton Opaque continued from 2 through to 4 years. While the rate of loss up to the 2 year review was greater for Helioseal compared to Delton Opaque, a component of the increased rate of wear for Helioseal was likely to be due to the increased area applied at baseline. It has been suggested that with larger amounts of sealant applied there is an associated greater early loss of material. ${ }^{14}$ As the mean area of Helioseal had reduced to become fractionally less than that of Delton Opaque at the 2 year review, the greater rate of peripheral wear of Helioseal from 2 through to 4 years can be considered to be a valid representation. The mean area reduction from 2 through to 4 years was greater for Helioseal compared to Delton Opaque at $1.23 \mathrm{~mm}^{2}$ and $0.81 \mathrm{~mm}^{2}$ respectively, the differences between the area reductions of these 32 matched pairs being statistically significant $(\mathrm{P}<.001)$. The loss of area throughout the 4 years in clinical service was over $99 \%$ attributed to wear. Resistance to wear has been suggested as an important factor regarding the durability of fissure sealants. ${ }^{24,25}$ Improving abrasion resistance has been considered potentially advantageous. ${ }^{26}$ With abrasive wear there is a reduction in both the thickness of a sealant and the area covered such that a point may be reached where a previously sealed fissure may be exposed. ${ }^{3}$ It has been stated that while properly applied sealant will last for many years, after many years sealant will wear down and selective loss can pose caries risk. ${ }^{27}$ Clinical trials over $4^{28}, 5^{29}, 7^{30}$, and 15 years ${ }^{31}$ demonstrate a progressive reduction in retention of unfilled Bis-GMA based fissure sealants over time. While reports of clinical trials measuring fissure sealant dimensions have demonstrated an initial rapid rate of loss, after which the rate slows, ${ }^{11-20}$ these studies have only been up to a maximum duration of 30 months. ${ }^{17}$ In the present study, the area of fissure sealant loss was demonstrated to be almost exclusively attributed to wear and after the initial rapid wear phase the peripheral wear was demonstrated to be progressive and not to plateau. This would support resistance to wear as being a more likely determinant of the longer term retention of unfilled Bis-GMA based fissure sealants.

\section{Conclusion}

The reported technique used optically clear replicas of fissure sealed teeth from a 4-year clinical trial and allowed their accurate sequential location and mounting in a predetermined plane of orientation. The use of a microscope with transillumination of the replicas from below with diffused light produced excellent definition of the periphery of the sealant and, in conjunction with digital imaging and computer graphic technology, enabled the systematic mapping of the peripheral border for all of the study sealants. The technique allowed the differentiation between and measurement of the area of peripheral material loss attributed to wear and bulk loss involving sealant-enamel interface failure. In addition, these areas of bulk loss were classified as either internal or external to the peripheral recession attributed to wear. The area of fissure sealant loss was demonstrated to be almost exclusively attributed to wear. The rate of peripheral wear of Helioseal was greater than for Delton Opaque. Irrespective of the material applied, after the anticipated initial phase of rapid wear, the peripheral recession attributed to wear was demonstrated to be progressive and not to plateau. Consequently, resistance to wear can be considered a more likely determinant of the longer term retention of unfilled Bis-GMA based fissure sealants.

\section{Acknowledgments}

None. 


\section{Conflicts of interest}

The authors declare there are no conflicts of interest.

\section{References}

1. Ripa LW. Occlusal sealants: rationale and review of clinical trials. Int Dent J. 1980;30(2):127-139.

2. Ripa LW. The current status of pit and fissure sealants. A review. J Can Dent Assoc. 1985;51(5):367-380.

3. Charbeneau GT. Pit and fissure sealants. Int Dent J. 1982;32:215-222.

4. Rock WP. Fissure sealants: Further results of clinical trials. $\mathrm{Br}$ Dent $J$ 1974;136(8):317-321.

5. Rock WP, Bradnock G. Effect of operator variability and patient age on the retention of fissure sealant resin: 3-year results. Community Dent Oral Epidemiol. 1981;9(5):207-209.

6. Going RE, Haugh LD, Grainger DA, et al. Four-year clinical evaluation of a pit and fissure sealant. J Am Dent Assoc. 1977;95(5):972-981.

7. Douglas WH, Tranter TC. A clinical trial of a fissure sealant-results after two years. Proc Br Paedod Soc. 1975;5:17-28.

8. Sundfeld RH, Mauro SJ, Briso ALF, et al. Clinical/Photographic evaluation of a single application of two sealants after eleven years. Bull Tokyo Dent Coll. 2004;45(2):67-75.

9. Davies NE, Tranter TC, Whitten JR. Evaluation of fissure sealant durability in vivo using an impression technique. J Dent. 1975;3(4):153156.

10. Handelman SL, Jensen OE, Pameijer CH. Quantitative assessment of sealant wear in vivo. J Prosthet Dent. 1978;40(5):531-533.

11. Jensen OE, Handelman SL, Pameijer CH. Clinical assessment of wear of two pit and fissure sealants. J Prosthet Dent. 1981;46(6):639-641.

12. Jensen OE, Handelman SL, Perez-Diez F. Occlusal wear of four pit and fissure sealants over two years. Pediatr Dent. 1985;7(1):23-29.

13. DeLong R, Pintado M, Douglas WH. Measurement of change in surface contour by computer graphics. Dent Mater. 1985;1(1):27-30.

14. Pintado MR, Conry JP, Douglas WH. Measurement of sealant volume in vivo using image-processing technology. Quintessence Int. 1988;19(9):613-617.

15. Conry JP, Pintado MR, Douglas WH. Measurement of fissure sealant surface area by computer. Quintessence Int. 1990;21(1):27-33.

16. Conry JP, Pintado MR, Douglas WH. Quantitative changes in fissure sealant six months after placement. Pediatr Dent. 1990;12(3):162-167.
17. Pintado MR, Conry JP, Douglas WH. Fissure sealant wear at 30 months: new evaluation criteria. J Dent. 1991;19(1):33-38.

18. Muhlbauer C, Herr P, Holz J, et al. Photometric method intended for clinically controlled evaluation of fissure sealants (Methode photometrique destinee au controle clinique des scellements de fissures). Rev mens suisse Odonto-stomatol. 1981;91:473-490.

19. Maeder A, Herr P, Holz J, et al. Controlled evaluation of clinical behaviour of 6 fissure sealant resins, after 2 years (Controle du comportement clinique de 6 resines de scellement de fissures apres 2 ans). Rev mens suisse Odonto-stomatol. 1982;92:1127-1133.

20. Tripodi D, Filippakos A, Piattelli A, et al. Wear of dental sealing materials using the replication technique. Eur J Paediatr Dent. 2011;12(2):95-98.

21. Niibu I, Tachikawa Y, Uji T, et al. Longitudinal observation on in vivo changes of pit and fissure sealant in the mouth. Part 1: Innovation of the method to standardize the three-dimensional position of resin replicas. The Japanese Journal of Pediatric Dentistry. 1985;23(4):917-925.

22. Mejare I, Mjor IA. Glass ionomer and resin-based fissure sealants: a clinical study. Scand J Dent Res. 1990;98(4):345-350.

23. Fernandes KS, Chalakkal P, Noronha de Ataide Id, et.al. A comparison between three different pit and fissure sealants with regard to marginal integrity. J Conserv Dent. 2012;15(2):146-150.

24. Raadal M. Abrasive wear of filled and unfilled resins in vitro. Scand $J$ Dent Res. 1978;86(5):399-403.

25. Galo R, Contente MMMG, Borsatto MC. Wear of two pit and fissure sealants in contact with primary teeth. Eur J Dent. 2014;8(2):241-248.

26. Silverstone LM. State of the art on sealant research and priorities for further research. J Dent Educ. 1984;48(2):107-118.

27. Simonsen RJ, Neal RC. A Review of the clinical application and performance of pit and fissure sealants. Aust Dent J. 2011;56(1):45-58.

28. Williams B, Laxton L, Holt RD, et al. Fissure sealants: a 4-year clinical trial comparing experimental glass polyalkenoate cement with a bis glycidyl methacrylate resin used as fissure sealants. $\mathrm{Br}$ Dent $\mathrm{J}$. 1996;180(3):104-108.

29. Shapira J, Fuks A, Chosack A, et al. A comparative clinical study of autopolymerized and light-polymerized fissure sealants: five-year results. Pediatr Dent. 1990;12(3):168-169.

30. Mertz Fairhurst EJ, Fairhurst CW, Williams JE, et al. A comparative clinical study of two pit and fissure sealants: 7-year results in Augusta, GA. J Am Dent Assoc. 1984;109(2):252-255.

31. Simonsen RJ. Retention and effectiveness of dental sealant after 15 years. J Am Dent Assoc. 1991;122(10):34-42. 